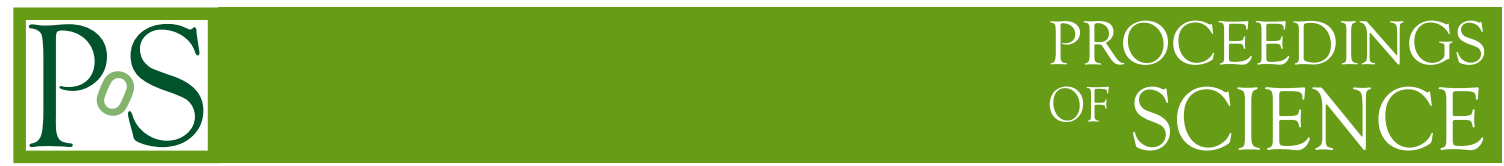

\title{
Searches for Dark Matter in ATLAS and CMS
}

\section{Dirk Zerwas* (on behalf of the ATLAS and CMS Collaborations)}

LAL, Univ. Paris-Sud, CNRS/IN2P3, Université Paris-Saclay, Orsay, France

E-mail: zerwaselal.in2p3.fr

CMS and ATLAS search for the presence of dark matter particles in missing transverse momentum $+\mathrm{X}$ final states, where $\mathrm{X}$ signals the production in association with jets, photons and gauge bosons. The results of these searches place stringent limits on the cross sections of interactions of dark matter with light and heavy flavor quarks and gluons. They are interpreted in terms of simplified models with different structures and mediators, as well as generic effective theory terms from higher mass scales.

The European Physical Society Conference on High Energy Physics 22-29 July 2015

Vienna, Austria

\footnotetext{
*Speaker.
} 


\section{Introduction}

The cosmic microwave background (e.g. Planck collaboration [1]) together with other probes and a cosmological model allows to determine the relic density [2]. Using a model that provides a dark matter particle candidate and its couplings, from which the dark matter annihilation cross section is derived, the relic density leads to a restriction of the allowed model parameter space (e.g. [5]).

The ATLAS [3] and CMS [4] experiments have searched for dark matter particles in protonproton collisions in center-of-mass energies of up to $8 \mathrm{TeV}$ in data corresponding to an integrated luminosity of up to $25 \mathrm{fb}^{-1}$ per experiment. While the indirect search via the relic density relies on the knowledge of the dark matter annihilation cross section, the search at the LHC is based on the pair production of dark matter particles from Standard Model particles. Assuming that the dark matter particles are massive and weakly interacting (WIMPs), they can only be detected by reconstructing the particles recoiling against them such as, for example, photons, jets and heavy gauge bosons. This is commonly denoted as the mono- $\mathrm{X}$ signature. Consequently the experimental challenge for these searches is the understanding of the distribution of the magnitude of the missing transverse momentum vector $E_{\mathrm{T}}^{\mathrm{miss}}$.

The results can be interpreted in several different ways. The first option is to derive a limit on the production cross section of the mono-X signature. The second possibility is to use Effective Field Theory (EFT) to translate the search for production of WIMPs into WIMP-nucleon scattering cross sections. This is particularly useful to compare the results of the LHC searches to those of the direct search for dark matter via WIMP-nucleon scattering. However this translation depends on the assumption of the effective operator to be used, e.g., see Ref. [6] for a classification of the operators. While EFT is an attractive option one has to be careful with the validity of the EFT results as these imply that the momentum transfer is small with respect to the (unkown) mediator mass. A third possibility is to use simplified models where the particle mediating the interaction between the Standard Model particles and the WIMPs is defined explicitly.

The production cross section depends on the mass of the dark matter candidate, its coupling and the suppression mass $M_{*}$. The suppression mass is $m_{V} / \sqrt{g_{f} g_{\chi}}$, where $m_{V}$ is the mass of the mediator of the production process and $g_{f}, g_{\chi}$ are the coupling strengths of the mediator to the Standard Model fermions and the dark matter candidate respectively.

\section{Searches and Results}

The search for the mono-photon signature by ATLAS and CMS is based on requiring large $E_{\mathrm{T}}^{\text {miss }}$ and a photon with large transverse momentum reconstructed in the central part of the detector $[7,8]$. To be precise ATLAS requires a photon of $125 \mathrm{GeV}$ and CMS a photon of $145 \mathrm{GeV}$, whereas the requirement on $E_{\mathrm{T}}^{\text {miss }}$ is $140 \mathrm{GeV}$ in CMS and $150 \mathrm{GeV}$ in ATLAS. Events with reconstructed leptons are vetoed, but events with one reconstructed jet are accepted.

Two different approaches are used to determine the background, mainly coming from events where a $\mathrm{Z}$ boson is produced and decays to a pair of neutrinos: ATLAS defines control regions where the signal is expected to be small and normalizes the background in these regions from the data. A further set of regions with low signal contamination is used to cross check the background 

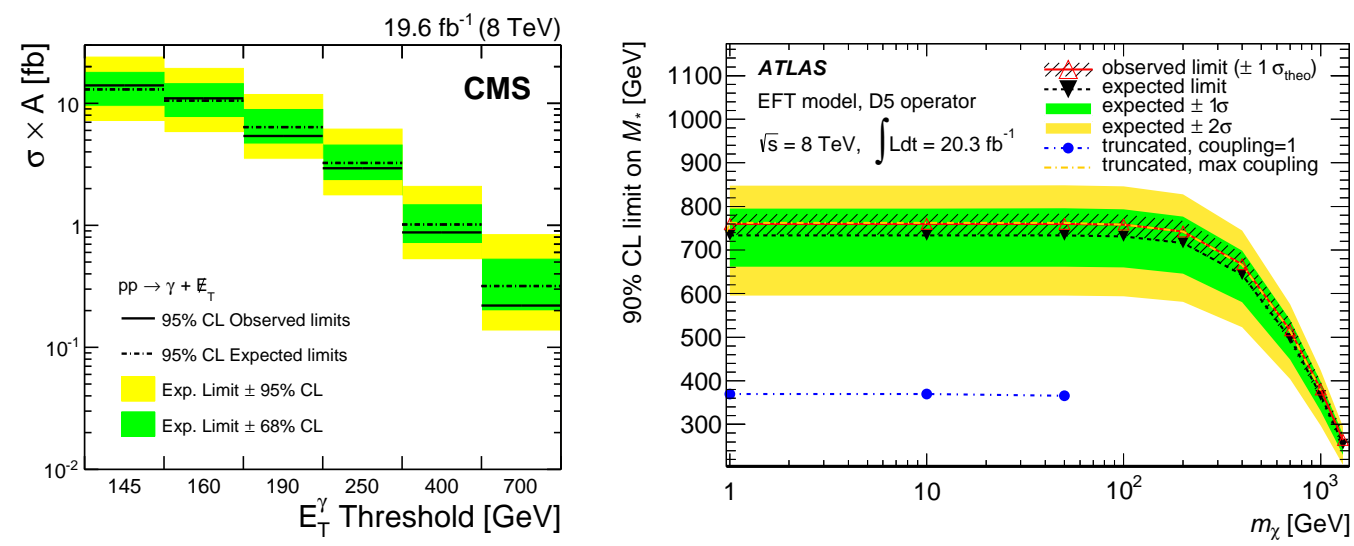

Figure 1: (Left) The 95\% CL cross section limits of the CMS mono-photon search is shown as function of the photon transverse energy threshold [8]. (Right) The 90\% CL limit on the suppression mass is shown as function of the WIMP mass for the D5 operator in the ATLAS mono-photon search with and without truncation [7].

estimation. CMS uses the Monte Carlo corrected for data/Monte Carlo differences for the estimation of the background. Control regions are used for a cross check of the estimation. In the ATLAS analysis 521 events are observed for $557 \pm 36 \pm 27$ expected, whereas in the CMS analysis 630 events are observed and $614 \pm 63$ are expected. As no significant deviations are observed, limits are calculated. In Figure 1(Left) the 95\% CL limit on the cross section times acceptance, determined by CMS, is shown. The limits depend on the threshold applied to the photon transverse energy. The result of the interpretation of the data in EFT for the D5 operator is shown in Figure 1(Right) by the ATLAS collaboration. Suppression masses up to almost $800 \mathrm{GeV}$ are excluded for a large range of WIMP masses. EFT is not a good approximation when the momentum transfer of the interaction is of the order of the mediator mass. The truncation method rejects simulated events where the momentum transfer is greater than the mediator mass. The mediator mass decreases when the coupling product $g_{f} g_{\chi}$ decreases. While for the maximal coupling the EFT limit is valid, for $g_{f} g_{\chi}=1$, the limit on the suppression mass decreases to roughly $380 \mathrm{GeV}$ when truncation is applied.

In the search for the mono-jet signature large $E_{\mathrm{T}}^{\text {miss }}$ and a central jet with a transverse momentum of at least $110 \mathrm{GeV}$ is required. The ATLAS collaboration defined nine signal regions which differ in the requirement on $E_{\mathrm{T}}^{\text {miss }}$ ranging from $150 \mathrm{GeV}$ to $700 \mathrm{GeV}$ [9] . In the region with the strongest requirement 126 events are observed for $97 \pm 14$ expected. The result of the CMS analysis requiring $E_{\mathrm{T}}^{\text {miss }}$ of at least $550 \mathrm{GeV}$ is the observation of 519 events where $509 \pm 66$ are expected [10]. The resulting limit on the suppression mass as function of the mediator mass is shown Figure 2(Left) for two simplified models which differ in the assumption on the WIMP mass $(50 \mathrm{GeV}$ and $400 \mathrm{GeV}$ ). If only EFT was used, the limit would not depend on the mediator. The figure shows that, depending on the value of the mediator mass, the EFT can over- and underestimate the limit on the suppression mass. In Figure 2(Right) the limits on the WIMP-nucleon scattering cross section are shown for several operators by CMS and compared to the results from the direct detection experiments. While the absolute value of the LHC limit depends on the as- 

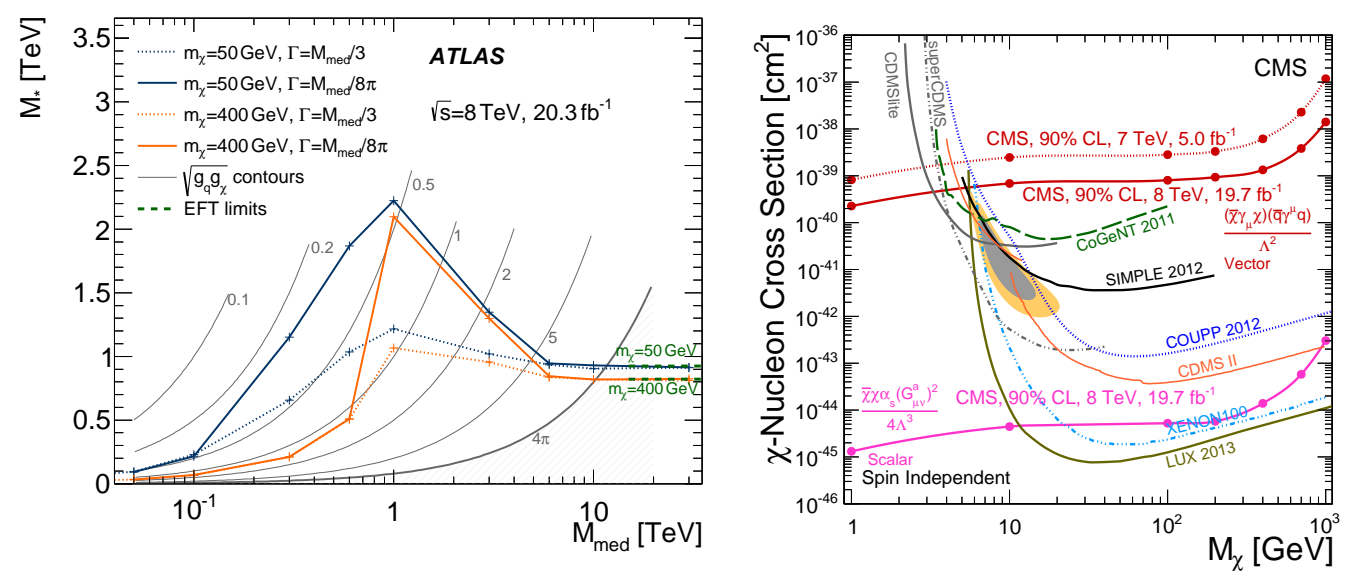

Figure 2: (Left) The limit on the suppression mass as function of the mediator mass is shown for the ATLAS mono-jet analysis for two WIMP masses [9]. (Right) The limit on the WIMP-nucleon scattering cross section is shown as function of the WIMP Mass for different operators for the CMS mono-jet analysis and compared to direct detection experiments [10].

sumption on the operator used to interpret the data, the common feature of all operators is that the LHC results are complementary to the direct searches at small WIMP masses.
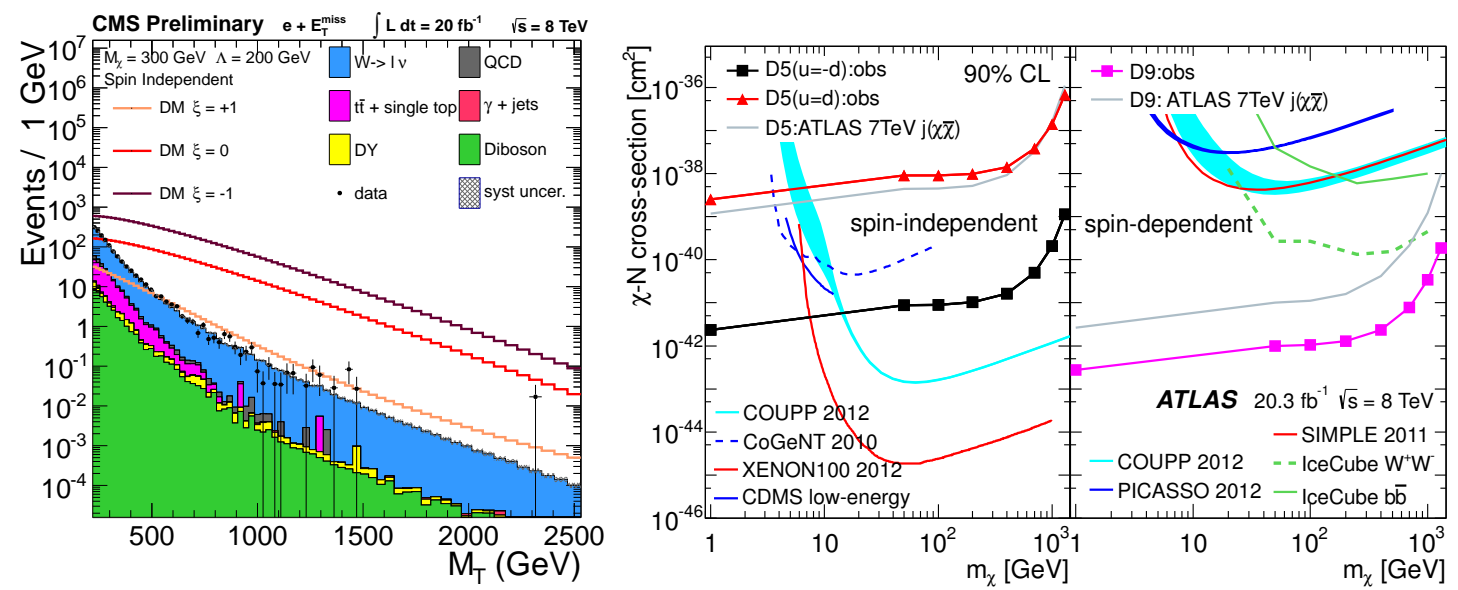

Figure 3: (Left) The transverse mass distribution is shown for the data and the background in the CMS mono-W analysis [12]. (Right) The limits on the spin-dependent and spin-independent WIMP-nucleon scattering cross sections are shown as function of the WIMP mass for the ATLAS mono-W analysis for different operators and different sign for the interference term [11].

The search for the mono-W signature by CMS in Ref. [12] relies on the leptonic decay of the W boson. The transverse mass, shown in Figure 3(Left) calculated from the transverse momentum of the reconstructed lepton and the transverse missing momentum, is used. Excellent agreement between the data and the background description is observed. For a requirement on the transverse mass of at least $1 \mathrm{TeV}, 22$ events are observed for $26 \pm 2.5$ expected in the electron channel and 33 events are observed for $33 \pm 26$ expected in the muon channel.

In the search for the mono-Z/W channel of ATLAS [11] the hadronic decays of the vector 

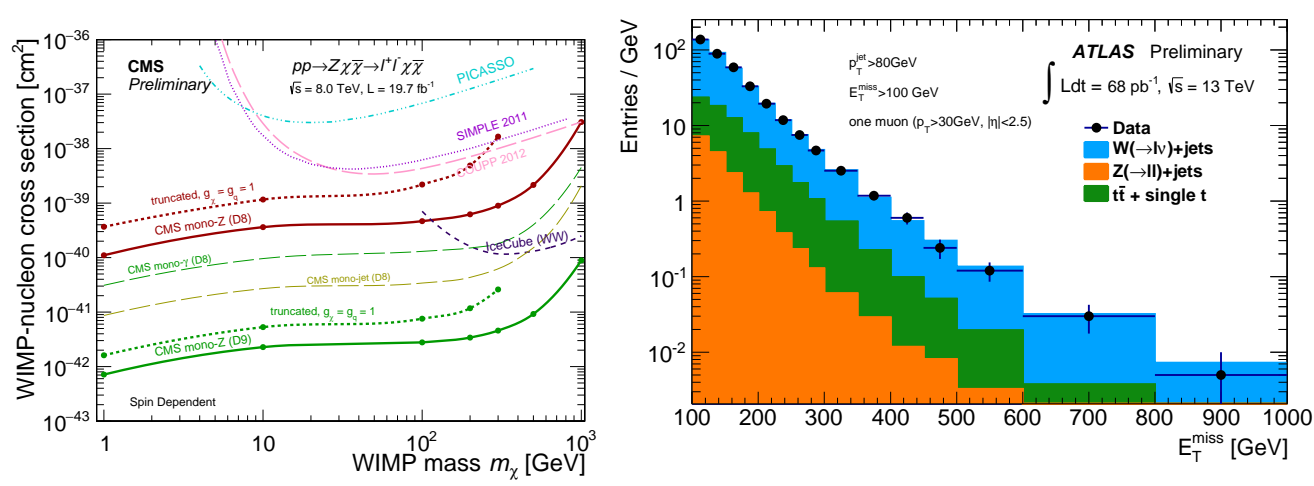

Figure 4: (Left) The limit on the WIMP-nucleon scattering cross section is shown as function of the WIMP mass for the CMS mono-Z analysis [13]. (Right) The distribution of $E_{\mathrm{T}}^{\text {miss }}$ is shown for an integrated luminosity of $68 \mathrm{pb}^{-1}$ recorded at a center-of-mass energy of $13 \mathrm{TeV}$ [15].

bosons are used. A central jet with a large radius parameter is required. If the electroweak vector boson is produced with a strong boost, the large jet will contain both jets from its hadronic decay. The analysis of the substructure of the large jet reduces the background to manageable level. For a requirement of at least $500 \mathrm{GeV} E_{\mathrm{T}}^{\text {miss }}, 89$ events are observed in agreement with $89_{-12}^{+9}$ expected. The resulting limits on the spin-dependent and spin-independent cross WIMP-nucleon scattering cross sections are shown in Figure 3(Right) as function of the WIMP mass. The partonic process of WIMP pair production is $d \bar{u} \rightarrow W \chi \chi$ and $u \bar{d} \rightarrow W \chi \chi$. After the emission of the $\mathrm{W}$ boson from the initial state $u$-quark, the process is $d \bar{d} \rightarrow \chi \chi$ while the partonic process is $u \bar{u} \rightarrow \chi \chi$ if the $\mathrm{W}$ boson is emitted from the $\bar{d}$-quark. With two diagrams the production cross section depends on the assumption of the strength of the effective coupling of the $u$-quark and of the $d$-quark to the dark matter particles and on the interference between the two diagrams. As shown in Figure 3(Right) for the D5 operator the limit improves when a negative relative sign is assumed between the two couplings.

The CMS collaboration has also searched for the mono- $Z$ signature using the leptonic decay of the $\mathrm{Z}$ boson and for a boosted signature of the hadronic decay of vector bosons [13, 14]. For the $\mathrm{Z}$ boson decay to electrons 111 events are observed, $113 \pm 4.0 \pm 12.5$ expected, for the decay to a pair of muons, 133 events are observed, $150.9 \pm 3.9 \pm 17.8$ expected in a good agreement with the background expectation. In Figure 4(Left) the limit on the WIMP-nucleon scattering cross section as function of the WIMP mass for the mono- $\mathrm{Z}$ search. The effect on the limit using the truncation technique is also shown for coupling strengths of $g_{f}=g_{\chi}=1$. The strongest limit is obtained with the D9 operator.

The data recorded at a center-of-mass energy of $13 \mathrm{TeV}$ has been analyzed in the control regions [15]. In Figure 4(Right) the distribution of $E_{\mathrm{T}}^{\text {miss }}$ is shown for the control region where the Standard Model background of $\mathrm{W}$ boson production is determined. The data sample corresponding to $68 \mathrm{pb}^{-1}$, after requiring a muon, a jet with at least $80 \mathrm{GeV}$ transverse momentum, shows good agreement between the data and the background expectation. To improve on the results obtained at 
$8 \mathrm{TeV}$, a larger data set is necessary.

The ATLAS/CMS dark matter forum has studied the potential of the LHC run starting this year in Ref. [16]. Taking the D5 operator with a dark matter particle mass of $50 \mathrm{GeV}$ as an example, with an integrated luminosity of only $5 \mathrm{fb}^{-1}$ the sensitivity in the suppression mass reaches $1.5 \mathrm{TeV}$, where the $8 \mathrm{TeV}$ data allowed to reach about $800 \mathrm{GeV}$.

\section{Conclusions/Outlook}

ATLAS and CMS have searched for dark matter via the recoil of photons, jets and electroweak bosons. As no evidence for dark matter has been observed, the results are presented as excluded domains in terms of effective operators, simplified models and translated into WIMP-nucleon scattering cross sections. At low dark matter mass, depending on the assumption of the operator used, the results are complementary to the searches by dedicated dark matter experiments.

\section{References}

[1] R. Adam et al. [Planck Collaboration], arXiv:1502.05956 [astro-ph.CO].

[2] P. A. R. Ade et al. [Planck Collaboration], Astron. Astrophys. 566 (2014) A54 [arXiv:1311.1657 [astro-ph.CO]].

[3] ATLAS Collaboration, JINST 3 (2008) S08003.

[4] CMS Collaboration, JINST 3 (2008) S08004.

[5] S. Henrot-Versillé, R. Lafaye, T. Plehn, M. Rauch, D. Zerwas, S. Plaszczynski, B. Rouillé d'Orfeuil and M. Spinelli, Phys. Rev. D 89 (2014) 5, 055017 [arXiv:1309.6958 [hep-ph]].

[6] J. Goodman, M. Ibe, A. Rajaraman, W. Shepherd, T. M. P. Tait and H. B. Yu, Phys. Rev. D 82 (2010) 116010 [arXiv:1008.1783 [hep-ph]].

[7] ATLAS Collaboration, Phys. Rev. D 91 (2015) 1, 012008 [Phys. Rev. D 92 (2015) 5, 059903] [arXiv:1411.1559 [hep-ex]].

[8] CMS Collaboration, arXiv:1410.8812 [hep-ex].

[9] ATLAS Collaboration, Eur. Phys. J. C 75 (2015) 7, 299 [Eur. Phys. J. C 75 (2015) 9, 408] [arXiv:1502.01518 [hep-ex]].

[10] CMS Collaboration, Eur. Phys. J. C 75 (2015) 5, 235 [arXiv:1408.3583 [hep-ex]].

[11] ATLAS Collaboration, Phys. Rev. Lett. 112 (2014) 4, 041802 [arXiv:1309.4017 [hep-ex]].

[12] CMS Collaboration, "Search for dark matter in the mono-lepton channel with pp collision events at center-of-mass energy of $8 \mathrm{TeV}$," CMS-PAS-EXO-13-004.

[13] CMS Collaboration, "Search for dark matter and unparticles produced in association with a $\mathrm{Z}$ boson in pp collisions at sqrt(s) $=8 \mathrm{TeV}$," CMS-PAS-EXO-12-054.

[14] CMS Collaboration, "Search for New Physics in the V-jet + MET final state," CMS-PAS-EXO-12-055.

[15] ATLAS Collaboration, https://atlas.web.cern.ch/Atlas/GROUPS/PHYSICS/PLOTS/EXOT-2015-005, EXOT-2015-005.

[16] ATLAS/CMS dark matter forum, arXiv:1507.00966 [hep-ex]. 